Check for updates

Cite this: RSC Adv., 2019, 9, 17737

\title{
Gelatin methacryloyl (GelMA)-based biomaterials for bone regeneration
}

\author{
Zhenqiang Dong, $\dagger^{\mathrm{a}}$ Qijuan Yuan, $\dagger^{\mathrm{b}}$ Keqing Huang, ${ }^{\mathrm{b}}$ Wanli Xu, ${ }^{\mathrm{a}}$ Guiting Liu ${ }^{* \mathrm{~b}}$ \\ and Zhipeng Gu (D) *bc
}

Gelatin methacryloyl (GelMA)-based biomaterials have been widely used in various biomedical applications due to their suitable biological properties and tuneable physical characteristics. In particular, GelMA can be used as a versatile matrix for bone tissue engineering scaffolds via various strategies to overcome major obstacles such as insufficient mechanical property and uncontrollable degradation. This review presents the research status of GelMA, its structure and function, GelMA-based biomaterials and the development of methods along with their existing challenges.

Received 10th April 2019

Accepted 14th May 2019

DOI: 10.1039/c9ra02695a

rsc.li/rsc-advances

possesses excellent self-healing ability, small size defects could

\section{Introduction}

Bone defects caused by physical injuries from accidents need effective treatments; as is known, natural bone tissue is a complex material composed of inorganic and organic composites with an elaborately hierarchical structure at macro-, micro- and nano-scales, which results in extremely high strength and toughness..$^{1-3}$ Moreover, the broadly distributed blood vessels in natural bone provide nutrition and clear waste to support tissue metabolism. For a healthy natural bone, which

${ }^{a}$ Department of Chemistry and Material Engineering, Quzhou University, Quzhou 324000, P. R. China

${ }^{b}$ Key Laboratory of Sensing Technology and Biomedical Instrument of Guangdong Province, School of Biomedical Engineering, Sun Yat-sen University, Guangzhou, 510006, P. R. China. E-mail: guzhp@mail.sysu.edu.cn; liugt3@mail.sysu.edu.cn

${ }^{`}$ Research Institute of Sun Yat-sen University in Shenzhen, Shenzhen, 518057, PR China

$\dagger$ These authors contribute equally to this work.

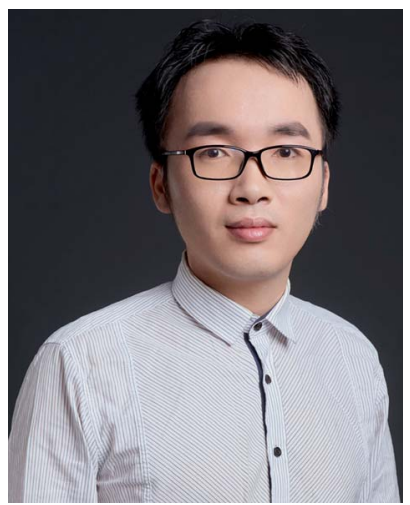

Zhipeng Gu graduated with a degree in Polymer Materials and Engineering in 2009 and received his $P h D$ in Material Science in 2014, both from Sichuan University. He was a postdoctoral researcher in the Department of Neurosurgery, West China Hospital, Sichuan University. He currently works in the Biomedical Engineering department at Sun Yat-sen University. His research aims to develop various polymeric hydrogels as different strategies for bone regeneration and wound healing. be mended over time without any extra management or surgeries. ${ }^{4}$ The healing process mainly refers to three steps: cartilage formation, biomineralization in the cartilage and bone formation. During this process, the related cells would be recruited to the defect with a specific microenvironment and growth factors, followed by proliferation and differentiation, which could lead to mineralization and ultimately regeneration of bone tissue. However, in the case of severe bone defects, the bone tissues cannot regenerate by themselves without extra surgical interventions. ${ }^{5,6}$

To deal with severe defects in bone tissues, autografts and allografts have been clinically utilized. ${ }^{7}$ However, these strategies could not absolutely meet clinical demands due to the insufficient supply of autografts and allografts. Therefore, the development of artificial implants, which can replace or regenerate bone tissues with appropriate mechanical properties, good biocompatibility and even excellent bioactivity, seems to be the only way to tackle the abovementioned issue.

In the past few decades, researchers have paid significant attention to artificial bone scaffolds. To date, a number of artificial scaffolds have been developed to deal with bone defects. ${ }^{8,9}$ These scaffolds can be simply classified as naturally derived materials or synthetic materials according to the source of the material; specifically, synthetic materials used in bone scaffolds mainly include polylactic acid, polyglycolic acid, poly(lactate-hydroxyacetic acid) and inorganic materials such as titanium alloy, biological ceramics, bioactive glasses and calcium phosphate. ${ }^{10}$ As is known, inorganic materials, such as calcium phosphate, often possess good biocompatibility and osteoconductive properties, which can be beneficial for bone regeneration. However, inorganic materials may be fragile with limited flexibility; this makes it difficult to use them under certain kinds of conditions. Moreover, synthetic organic materials, such as polylactic acid, can be facilely synthesized with 
desired mechanical properties and good biocompatibility. However, synthetic organic materials usually lack bioactivity, which can be important for inducing bone regeneration. Thus, as another kind of promising materials, naturally derived materials have drawn the attention of researchers for the fabrication of bone regeneration scaffolds with good biocompatibility and low cytotoxicity. ${ }^{11}$

According to previous studies, naturally derived materials, such as collagen, fibrous protein, alginate and chitosan, have been widely utilized for bone regeneration. Among them, gelatin, a denatured product of collagen, has attracted significant attention of the researchers because of its natural Arg-GlyAsp (RGD) sequences, which can facilitate biological interaction between cells and scaffolds, and its matrix metalloproteinase sensitive moieties, which can lead to the degradation of collagen. ${ }^{12}$ Due to its low antigenicity, gelatin seems to be a promising material for the construction of scaffolds for bone regeneration, and it has been broadly used to fabricate tissue engineering scaffolds. ${ }^{13}$ However, its poor mechanical rigidity and uncontrollable degradation rate limit its application, especially for the treatment of loading burden bone defects. To solve this problem, researchers have modified gelatin with methacryloyl to graft unsaturated bounds onto the molecular chains. ${ }^{14-16}$ The resultant gelatin methacryloyl (GelMA), could photo crosslink in the presence of a photoinitiator, forming three-dimensional (3D) structures under the radiation of UV light. The crosslinked GelMA with excellent and controllable mechanical properties could match the requirements for scaffolds under diverse conditions. To date, GelMA-based biomaterials have been widely studied for their physical and biochemical properties with multitudinous applications ranging from drug delivery to tissue engineering; however, GelMA-based biomaterials have not been fully excavated in the field of bone tissue engineering. Herein, this review focused on the development of GelMA as a bone regeneration strategy, specifically GelMA-based biomaterials. Moreover, the challenges and prospect of GelMA-based biomaterials in the bone tissue engineering fields have been discussed.

\section{Structure and function of GelMA}

As above mentioned, gelatin, the denatured product of collagen, is hydrophilic, whereas its aqueous solution can form a hydrogel under environmental stimuli such as temperature, $\mathrm{pH}$ and multivalent cations; $;^{17-19}$ these kinds of physical or ionic crosslinked gelatin hydrogels are usually cured by the formation of a chain-assembled electrostatic interaction or metal-organic coordination; as a gelatin derivative containing many methacrylamide groups and few methacrylate groups, GelMA undergoes photoinitiated radical polymerization to form covalently crosslinked hydrogels. Due to its simple and controllable fabrications, it is widely used in biomedical applications with semi-industrial management. ${ }^{20}$ The modification route for GelMA is summarized in Fig. 1. As shown in the schematic, unsaturated bounds were grafted onto gelatin by the conversion of an anhydride to an amino and hydroxyl group via a simple one-step reaction.
A
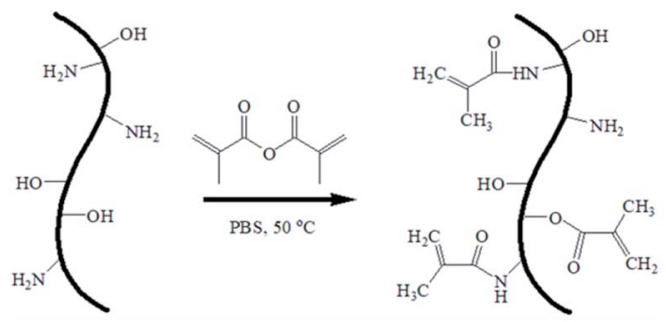

B

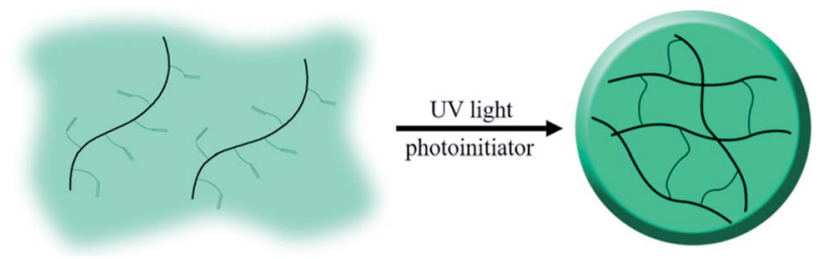

Fig. 1 Schematic of GelMA and the fabrication of its hydrogels.

The substitution of methacryloyl is hard to determine because of the complex components of gelatin with eighteen kinds of amino acids. To further control the mechanical properties and morphology of the GelMA hydrogels, several methods have been developed to quantify the substitution of methacryloyl; one of the strategies to determine the substitution is the use of ${ }^{1} \mathrm{H}$ NMR to evaluate the change in the lysine amino group after the reaction. ${ }^{21,22}$ This method could only calculate the methacrylamide groups during the grafting procedure, including both the amidation of the amino group and the esterification of the hydroxyl group, thus suggesting an underestimation of the gelatin methacryloyl substitution; moreover, several other methods could estimate the total substitution of methacryloyl but could not distinguish between the methacrylamide and methacrylate groups.$^{14}$ Recently, Southan et al. conducted 2D NMR experiments to derive detail information on the signals present in the ${ }^{1} \mathrm{H}$ NMR spectrum. ${ }^{23}$ Via this method, methacrylamide from the methacrylate groups as well as unbound impurities, such as methacrylic acid, could be identified. This quantification method provided a possibility to precisely control the substitution of GelMA, further regulating the property of hydrogels to match the requirements of scaffolds in bone regeneration. Furthermore, Kirsten Borchers investigated the influence of the substitution of methacryloyl in GelMA hydrogels. ${ }^{21}$ They found that higher methacrylation of GelMA led to lower viscosity of the precursor GelMA aqueous solution, which would be suitable in 3D bioprinting; otherwise, lower methacrylation of GleMA resulted in higher viscosity of the precursor GelMA aqueous solution, which could easily clog the printing nozzles and be hard to use in bioprinting; the chemically crosslinked GelMA hydrogel with enhanced mechanical rigidity, improved stability and multiparous architecture might be used as a suitable supporter in bone regeneration.

As is known, the double bonds in the GelMA could easily conjugate together intermolecularly, forming a 3D architecture as desired from a few seconds to several minutes. On the other hand, the GelMA content in hydrogels could also influence their 
properties during cell culture; to determine the appropriate formula of GelMA needed to construct an optimal scaffold suitable for cell adhesion, proliferation, and spreading, the influence of the GelMA concentration on the properties of hydrogels was investigated. ${ }^{24}$ The scaffolds with $5 \%$ and $10 \%$ concentration of GelMA were evaluated in terms of their porosity, pore size, swelling ability and mechanical properties. The results indicated that the scaffolds with GelMA at a 5\% concentration seeded with mesenchymal stem cells showed higher ability to promote extracellular matrix calcification as compared to the scaffolds with GelMA at a $10 \%$ concentration. They proposed that this result may have been caused by the higher porosity and pore size of the resulting hydrogel with low GelMA concentration in vitro. Moreover, Chen et al. investigated the influence of different levels of stiffness in the GelMA hydrogels on the chondrocyte phenotype by controlling the methacrylation degree of GelMA; ${ }^{25}$ they monitored the cell viability, cell morphology, and preservation of the chondrogenic phenotype after seeding the cells in the GelMA hydrogels with different substitutions of double bonds. The results showed that higher stiffness of hydrogels could benefit the preservation of the chondrogenic phenotype. These basic studies on the mechanical properties of the GelMA hydrogel and the relationship between the cell behavior and the mechanical properties of GelMA allowed researchers to construct GelMA hydrogel scaffolds with appropriate mechanical properties for effective bone regeneration. Compared to the physically crosslinked gelatin hydrogels, the chemically crosslinked gelatin methacryoyl hydrogel showed outstanding mechanical properties and good stability, which would be easy to handle during wound treating. This strategy not only increased the mechanical properties of the hydrogel, but also maintained the bioactivity of gelatin. Moreover, the GelMA hydrogel scaffolds with tuneable mechanical properties and biodegradation speed showed significant potential in bone regeneration.

\section{GelMA-based hybrid biomaterials}

Bone tissue is highly intricate such that a simply developed hydrogel without any additions or designations will not match the requirements for bone regeneration. In the bone tissue, the structure is anisotropic and varies at macro, micro and nanoscales. ${ }^{26}$ In the microenvironment of natural bone, different kinds of cells (osteocytes, osteoblasts, and osteoclasts) are incorporated into the hierarchical architecture. The special dynamic microenvironment composed of collagen, inorganic nanoparticles and growth factors could regulate the behavior of cells, guiding cell adhesion and differentiation. Blood vessels distributed in bone tissue supply nutrition and clear waste. The complexity of natural bone has drawn the attention of some researchers towards the fabrication of biomimetic gelatin scaffolds with a suitable biomechanical structure and physiology properties for bone regeneration. ${ }^{27,28}$ Inspired by the structure of natural bone tissue, they have produced scaffolds by introducing inorganic composites, growth factors, and even cells into the gelatin scaffolds to mimic the structure of the bone extracellular matrix.

\section{GelMA/inorganic biomaterial hybrid hydrogels for bone tissue} engineering

To mimic the components and structures of bone tissues, various inorganic substances were integrated into the hydrogels to enhance the functionality of GelMA hydrogel scaffolds for bone regeneration. For instance, Akhilesh K. Gaharwar designed an inorganic nanoparticle-enhanced composite GelMA hydrogel with ultralow concentration of nanoparticles. ${ }^{29}$ In this strategy, the modified iron oxide $\left(\mathrm{Fe}_{3} \mathrm{O}_{4}\right)$ magnetic nanoparticles (MNPs) were encapsulated into the GelMA hydrogels during the photo crosslinking process of the GelMA solution. As a result of the incorporation of MNPs, more than a 10-fold increase in the mechanical stiffness and a 20-fold increase in toughness were observed. Furthermore, the results indicated that the formation of imide bonds and the presence of carboxylate-amine interactions between the MNPs and the GelMA polymer played a vital role in the enhancement of the mechanical stiffness; the MNPs led to dual chemical crosslinking of the points interacting with the GelMA polymer chains from which polymeric cross-linking originated; this favoured bone regeneration.

Il Keun Kwon and his co-workers developed an approach for bone tissue regeneration. ${ }^{30}$ They constructed a scaffold based on a biodegradable gelatin methacryloyl hydrogel loaded with gold nanoparticles. The results showed that the GelMA modification in the scaffolds could promote the proliferation, differentiation, and the alkaline phosphate (ALP) activities of human adipose-derived stem cells (ADSCs). In the presence of GelMA-modified gold nanoparticles, human adipose-derived stem cells (ADSCs) could differentiate into osteoblast cells. The in vitro results also showed that GelMA-modified gold nanoparticles at high concentration could promote new bone formation. Akhilesh K. Gaharwar fabricated a bone scaffold by introducing nanosilicates into the GelMA hydrogel. ${ }^{31,32}$ The compressive modulus of these hydrogel composites increased 4-fold as compared to that of collagen-based hydrogels. The results indicated that nanosilicates containing a GelMA hydrogel could promote osteogenesis, even without related growth factors. The enhanced alkaline phosphatase activity and mineralization show that the nanosilicates containing GelMA hydrogels have prospects in bone regeneration. In another study, Liang Chen grafted mesoporous bioactive glass nanoparticles into GelMA by an amide reaction to construct an artificial periosteum..$^{33}$ This nanocomposite hydrogel also exhibited improved angiogenesis and osteogenesis properties along with better mechanical properties. In a similar strategy, bioactive hollow particles were added to form a nanocomposite GelMA hydrogel. ${ }^{34}$ The addition of porous nanoparticles enhanced the resistance of the hydrogels to swelling due to the high surface area of porous nanoparticles. The in vivo experiments showed that this hollow nanoparticle-enhanced GelMA hydrogel scaffold could promote the healing of large bone defects $5 \mathrm{~mm}$ in diameter. Xingdong Zhang integrated hydroxyapatite into GelMA; this resulted in the formation of a double-ring structure-encapsulated human umbilical vascular endothelial cells in the inner ring and human osteoblast-like 
cells in the outer ring. ${ }^{35}$ The enhanced genic expression of the osteogenesis-related and angiogenesis-related differentiations during the coculture of the two kinds of cells could be detected. Nano hydroxyapatite could also be used as an inducer to enhance the additives and osteogenesis in the GelMA hydrogel. ${ }^{36}$ Graphene oxide encapsulated in photo-crosslinked GelMA hydrogels has also been determined to promote the chondrogenic differentiation of hMSCs as compared to GelMA hydrogels without the addition of graphene oxide using 3D printing technology. ${ }^{37}$

Thus, based on previous studies, we can conclude that inorganic components play a vital role in bone regeneration by regulating the mechanical properties and cell differentiation process $;^{38}$ however, the dispersion of inorganic components in the GelMA hydrogels needs additional consideration.

\section{GelMA/organic biomaterial hybrid hydrogels for bone tissue engineering}

Apart from the addition of inorganic components, some biodegradable organic materials have also been utilized to introduce some other specific functions into the GelMA scaffolds. In 2017, Zhang et al. used polylactic acid to introduce a 3D printed microstructure into the GelMA hydrogels via fused deposition modeling for bone regeneration. ${ }^{39}$ The compressive modulus of the polylactic acid-reinforced GelMA hydrogel was even much higher than the modulus of mandibular bone.

On the other hand, some researchers have taken advantages of the extracellular matrix, which has a structure very close to that of natural tissues. They have proposed that the addition of a natural extracellular matrix may facilitate bone regeneration. Malda et al. developed a GelMA hydrogel entrapped with cartilage-derived matrix (CDM) particles. ${ }^{40}$ The in vitro experiments showed that the CDM particles could stimulate the differentiation of MSCs co-entrapped in the GelMA hydrogels and promote the formation of cartilage. This method provided another direction towards the fabrication of scaffolds for bone regeneration. By our group, black phosphorus-incorporated GelMA/cationic arginine-based unsaturated poly(ester amide) hybrid hydrogel scaffolds have been investigated for enhancing bone regeneration. In vitro and in vivo results indicated that the GelMA/organic biomaterial hybrid hydrogels could be an effective strategy for bone regeneration. ${ }^{41}$

\section{GelMA hydrogels with growth factor for bone tissue engineering}

Since bone regeneration is a complex process, by only considering the mechanical properties of scaffolds, the requirements of bone regeneration cannot be met; the biological properties of GelMA scaffolds have a significant influence on the bone regeneration process since cell adhesion, proliferation, spreading and differentiation can be controlled by the bioactivity of scaffolds in an ambient microenvironment. Many researchers have paid significant attention to improve the biological function of scaffolds. ${ }^{42}$ Moreover, osteogenic and angiogenic growth factors, such as bone morphogenetic protein (BMP) and vascular endothelial growth factor, have been introduced into the GelMA hydrogels along with organic or inorganic reinforcement components.$^{\mathbf{4 3 , 4 4}}$ These specially designed GelMA hydrogel scaffolds with increased bioactivity and mechanical properties exhibited better performance in bone regeneration. Although the incorporation of growth factors can promote angiogenesis and osteogenesis in scaffolds at defects, it has not been widely utilized. Moreover, some researchers have investigated new methods to improve the biological properties without the use of growth factors. This may be because the incorporation of growth factors is consumptive, and the activity of growth factors is hard to maintain for a long time.

\section{GelMA hydrogels containing cells for bone tissue engineering}

To the best of our knowledge, tissue engineering scaffolds cultured with cells can provide a more direct approach to promote bone tissue regeneration. Therefore, Weitz et al. have incorporated bone marrow-derived mesenchymal stem cells (BMSCs) into GelMA using a microfluidics-assisted technology to increase cell retention after injection into bone tissues. ${ }^{45}$ They found that osteogenesis was enhanced after entrapping the BMSCs in a photo-crosslinked GelMA microgel. The increased mineralization can further demonstrate the promotion of bone regeneration.

Khademhosseini and co-workers designed a GelMA-based scaffold entrapped with both osteogenic and angiogenic cells. ${ }^{\mathbf{4 6 , 4 7}}$ The diligent design successfully led to simultaneous mineralization and angiogenesis. Remarkably, angiogenesis in the scaffold could further promote cell proliferation and spreading that further accelerated bone regeneration.

\section{GelMA as the surface-modified biomaterials}

Under some special conditions, such as the loss of large scale bone tissue or dental restorations, the GelMA scaffolds can be used as a coating on the surface of some prostheses to promote their interaction with the host bone. Surface functionalization of this prosthesis to prevent post-surgery infection and promote biointegration is often used for long-term success after implantation. GelMA was used as a kind of bioactive material to decorate metal prosthesis before implantation. In one of the methods, silicate nanoparticles and cationic antimicrobial peptides were entrapped in the GelMA hydrogel function membrane to enhance osseointegration and reduce infection, respectively. ${ }^{48}$ In addition, dopamine was chemically conjugated to the GelMA molecule to improve its adhesion to the surface of metal implants; in this strategy, GelMA acted as a bridge between native bone tissue and prosthesis, preventing loss after implantation by osteogenesis in the GelMA hydrogel.

\section{Construct method for GelMA-based hybrid biomaterials}

Bone tissues have a hierarchical structure at macro, micro and nano scales, composing of cortical bone, spongy bone and blood vessels that supply nutrients and maintain cell viability to maintain the bone tissue bioactivities and self-regenerating 
ability. ${ }^{49}$ The special architecture and composition of bone tissue provides biological activity along with its excellent stiffness and toughness as a load-bearing tissue. Once defects formed under the damage from external stress, adjacent cells could be recruited to proliferate, migrate, differentiate, and further secrete collagen and silicate nanoparticles along the collagen fibers. Finally, new bone tissue formed to repair the defects in response to external stress-caused damages to physical and biological integrity as compared to the original bone tissue. For defects beyond the crucial size, the bone tissue cannot regenerate via self-regulation of inner cells and growth factors under the special damaged condition. After years of investigation, it has been proposed that the hierarchical structure and encapsulated cells and growth factors may contribute to the fast self-regeneration of damaged bone tissues. Based on these assumptions, an extra construction method is needed to build a mimetic microstructure of GelMA-based biomaterials for bone regeneration. To date, various construct methods, such as 3D printing, electrospinning, microfluidic technologies, etc., have been developed for the fabrication of scaffolds, including GelMA-based biomaterials. ${ }^{\text {50-53 }}$

\section{D printing}

Due to the development of manufacturing technology, there have been an increasing number of methods used to construct complicated structured scaffolds that incorporate manufacturing technologies; one of the most utilized methods for the fabrication of tissue engineering scaffolds is 3D technology. ${ }^{51}$ This fast gelation ability matches the requirements of the popularly used $3 \mathrm{D}$ printing technology. ${ }^{54}$ Once the bioprinting solution extrudes from the machine, it can be cured rapidly under UV light radiation focused on the formation position (Fig. 2). By mediating the intensity of the UV light, the substitution and concentration of GelMA, the addition of other components as well as the gelating speed could be slightly regulated according to machine's conditions. ${ }^{55}$ Apart from the gelating speed, the mechanical property could also be regulated, which was introduced above. Via the incorporation of the 3D bioprinting technology, a series of versatile porous structured scaffolds were fabricated with varying porous parameters.

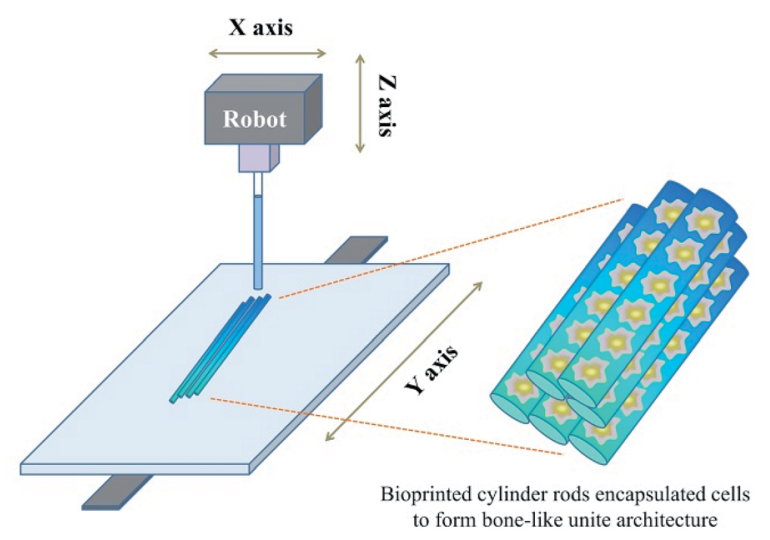

Fig. 2 Fabrication of the bone mimetic 3D architecture using the 3D bioprinted method to construct a bone-like unite architecture.
Recently, GelMA has been often combined with 3D printing to construct predesigned architectures for complex bone regeneration. This method required the GelMA solution to match the requests of the $3 \mathrm{D}$ printing; one important parameter was the viscosity of the precursor solution. A suitable viscosity ensures the successful gelation of GelMA, prevents the collapsing of extrusive GelMA before curing, and maintains the viability of entrapped cells. Sometimes, the simple regulation of the concentration or substitution of GelMA does not generally meet requirements. To address the abovementioned challenges, some organic materials have been utilized to mediate its properties. Among them, alginate, as one of materials possessing high viscosity in its aqueous solution, can self-cure instantaneously when exposed to calcium ions. ${ }^{56}$ Researchers took advantage of this property and utilized alginate to regulate the viscosity of the GelMA solution that ensured the stability of the architecture before photo-crosslinking.

Hairui Suo et al. utilized GelMA as a bioink for the fabrication of scaffolds via 3D bioprinting. ${ }^{57}$ They added unmodified gelatin, which can reversibly form hydrogels by changing the environmental temperature to regulate the processability of the GelMA bioink at low concentrations. A temperature controller was designed around the syringe of the $3 \mathrm{D}$ printing equipment with a cooling system under the receiving platform. The temperature controller ensured that the GelMA/gelatin bioink was a homogeneous solution. After being extruded onto the receiving platform, the solution became solid with a designed porous structure until it cured under the radiation of UV light; this prevented the transformation of the porous structure before the chemical crosslinking of GelMA. The physically crosslinked gelatin component could gradually dissolve when the temperature increased above the gel point of gelatin. They indicated that the loss of gelatin had no effect on the scaffold geometry. The biocompatibility of the GelMA/gelatin hybrid bioink was determined via cell morphology and viability tests after 3D printing of this cell-laden GelMA/gelatin bioink.

\section{Micromachining method}

For bone regeneration, not only biomaterials, but also the surface micro-topological structure can influence the regeneration process and functional construction of bone tissue. The inability of large bone defects to self-regenerate after damage is partially due to the destruction of the local vascular network, which supports nutrition during bone regeneration. Considering this remarkable role of the vasculature in bone tissue, researchers have attempted to construct a vascular structure in artificial scaffolds using a micromachining method. Ali Khademhosseini and his co-workers simultaneously introduced angiogenic and osteogenic cells into GelMA scaffolds with a predesigned micropattern to enhance the bone regeneration efficiency at defect sites. ${ }^{\mathbf{4}}$ In their studies, they constructed HUVEC/hMSC-encapsulated parallel angiogenic niches in osteogenic niches, as shown in Fig. 3. With the help of glass slides, which were treated with 3-(trimethoxysilyl) propyl methacrylate (TMSPMA) capable of grafting GelMA hydrogel on it via co-radiation from UV light, ultrathin hydrogels were 

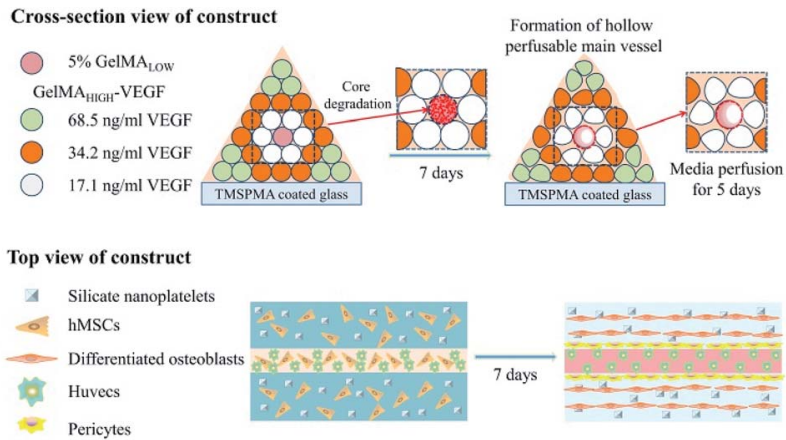

Fig. 3 Fabrication of the bone mimetic 3D architecture using the micromachining method to construct osteogenic and vasculogenic niches.

obtained that possessed a designed micropattern formed for further in vitro investigation. The micropatterned GelMA hydrogel was constructed by two steps. First, a GelMA 5\% (w/v) solution containing GFP-HUVECs and hMSCs at defined cell densities was added on a Petri dish and covered with a TMSPMA-treated glass slide. A photomask was covered on the top of glass slide. Thereafter, it was exposed to UV light for 35 seconds. The angiogenic niche was constructed in the form of an organized array by the photo-crosslinking of GelMA. Second, the osteogenic niche formed was overlaid on the micropatterned GelMA on the TMSPMA glass via the gelation of preosteoblast-labelled GelMA under UV exposure using the same gelation principle. The spaces between parallel-lined angiogenic niches were filled with the osteogenic niche for the formation of new bone tissue after the participation of preosteoblasts. The inner angiogenic niches could promote angiogenesis in the scaffolds in the presence of HUVECs and hMSCs. The angiogenic-related CD31 and $\alpha$-SMA expression were assessed to evaluate the angiogenic activity of the micropatterned scaffolds. The mineralization of the scaffold was also assessed with Alizarin Red in vitro. The result suggested that the osteogenesis of the GelMA hydrogel scaffolds was enhanced with the vasculature distributed in the form of predesigned arrays. Therefore, the conclusion can be made that the construction of the micropatterned GelMA scaffolds with precisely designed angiogenetic and osteogenic niches exhibit better performance as compared to the simple mixture of different components.

In another study reported by Khademhosseini, along with the purpose of fabricating vascularized scaffolds for bone regeneration, an extrusion-based direct-writing bioprinting strategy was developed to construct a bone-like tissue microstructure. ${ }^{47}$ As shown in Fig. 4, the central cylinder of the construct was printed with a low concentration of GelMA, which had a relative faster degradation rate. Blood vessels formed in the central cylinder with the degradation of the GelMA hydrogel of low concentrations. Around the central vascular cylinder, nanoplatelet-loaded GelMA hydrogels with relatively higher concentration as compared to those in the central cylinder were printed to promote osteogenesis around the central blood vessel; to enhance the spreading of vascular extensions towards

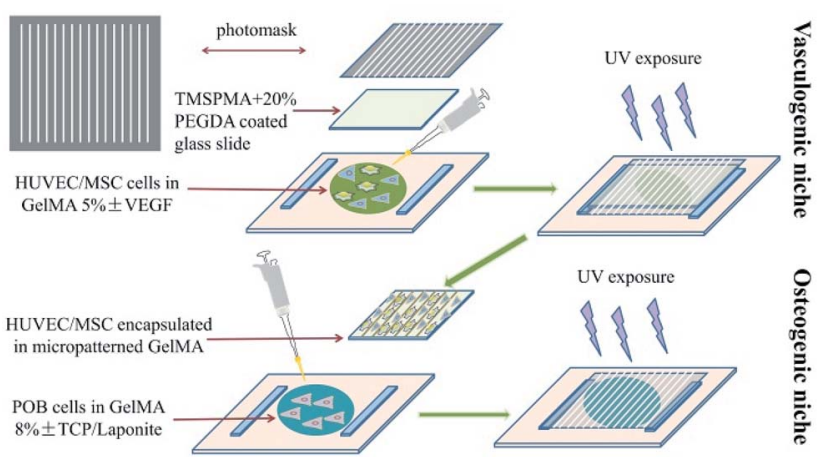

Fig. 4 The application of the micromachining method for the engineering of hydrogels to create 3D vascularized bone tissues.

the surrounding osteogenesis region, VEGF was chemically conjugated to GelMA with gradient concentration according to the distance from the central blood vessel. After 5 days of static culture, the cell in the osteogenic niches maintained their viability due to the nutrient support from the central vessel. Moreover, the co-culture of the hMSCs and HUVECs led to promoted angiogenesis.

\section{Microfluidic-assisted technology}

David Weitz and Wenguo Cui combined a minimally invasive method with tissue engineering scaffolds to simplify the engraftment process and settle the low cell retention after transplantation..$^{45}$ Microfluidic-assisted technology was utilized for the formation of GelMA hydrogel microspheres. The celllabeled microspheres were injected into the damaged position to investigate the bone regeneration efficiency. This strategy may simplify the operation process and maintain cell viability, which benefits from the space between microspheres and their contribution to nutrient/waste exchange; the shortcoming is that the microspheres cannot be used in the bear-loading or facilely deformed domains because of the amorphous microsphere suspension.

\section{Conclusion and perspective}

In summary, the development of GelMA-based biomaterials for tissue engineering, especially for bone tissue engineering, is still incomplete, and improvement is needed for many problems related to the mechanical properties, degradation rate, structure, morphology, as well as biological activity of these biomaterials; furthermore, with the development of osteoimmunology, systematic studies on immunology and some other activities of GelMA-based biomaterials would be required for the clinical applications of these biomaterials.

\section{Conflicts of interest}

There are no conflicts to declare. 


\section{Acknowledgements}

We sincerely acknowledge the funding and generous support of the National Natural Science Foundation of China (No. 5150030109) and the Fundamental Research Funds for the Central Universities (No. ln18lgpy58).

\section{Notes and references}

1 Y. Zheng, K. Huang, X. You, B. Huang, J. Wu and Z. Gu, Int. J. Biol. Macromol., 2017, 104, 1143-1149.

2 Z. Hao, Z. Song, J. Huang, K. Huang, A. Panetta, Z. Gu and J. Wu, Biomater. Sci., 2017, 5, 1382-1392.

3 S. Almubarak, H. Nethercott, M. Freeberg, C. Beaudon, A. Jha, W. Jackson, R. Marcucio, T. Miclau, K. Healy and C. Bahney, Bone, 2016, 83, 197-209.

4 Z. Gu, H. Xie, L. Li, X. Zhang, F. Liu and X. Yu, J. Mater. Sci.: Mater. Med., 2013, 24, 1251-1260.

5 S. Bose, M. Roy and A. Bandyopadhyay, Trends Biotechnol., 2012, 30, 546-554.

6 M. M. Stevens, Mater. Today, 2008, 11, 18-25.

7 S. S. Jensen and H. Terheyden, in Database of Abstracts of Reviews of Effects (DARE): Quality-assessed Reviews [Internet], Centre for Reviews and Dissemination, UK, 2009.

8 X. Wang, S. Xu, S. Zhou, W. Xu, M. Leary, P. Choong, M. Qian, M. Brandt and Y. M. Xie, Biomaterials, 2016, 83, 127-141.

9 M. Saini, Y. Singh, P. Arora, V. Arora and K. Jain, World Journal of Clinical Cases: WJCC, 2015, 3, 52.

10 A. Huang, X. Peng, L. Geng, L. Zhang, K. Huang, B. Chen, Z. Gu and T. Kuang, Polym. Test., 2018, 71, 101-109.

11 T. Gong, J. Xie, J. Liao, T. Zhang, S. Lin and Y. Lin, Bone Res., 2015, 3, 15029.

12 X. Liu, L. A. Smith, J. Hu and P. X. Ma, Biomaterials, 2009, 30, 2252-2258.

13 W. Jia, P. S. Gungor-Ozkerim, Y. S. Zhang, K. Yue, K. Zhu, W. Liu, Q. Pi, B. Byambaa, M. R. Dokmeci and S. R. Shin, Biomaterials, 2016, 106, 58-68.

14 J. W. Nichol, S. Koshy, H. Bae, M. H. Chang, S. Yamanlar and A. Khademhosseini, Biomaterials, 2010, 31, 5536-5544.

15 B. H. Lee, N. Lum, L. Y. Seow, P. Q. Lim and L. P. Tan, Materials, 2016, 9, 797.

16 H. Shirahama, B. H. Lee, L. P. Tan and N.-J. Cho, Sci. Rep., 2016, 6, 31036.

17 X. Zhao, Q. Lang, L. Yildirimer, Z. Y. Lin, W. Cui, N. Annabi, K. W. Ng, M. R. Dokmeci, A. M. Ghaemmaghami and A. Khademhosseini, Adv. Healthcare Mater., 2016, 5, 108118.

18 S. Das, F. Pati, Y.-J. Choi, G. Rijal, J.-H. Shim, S. W. Kim, A. R. Ray, D.-W. Cho and S. Ghosh, Acta Biomater., 2015, 11, 233-246.

19 S. T. Koshy, R. M. Desai, P. Joly, J. Li, R. K. Bagrodia, S. A. Lewin, N. S. Joshi and D. J. Mooney, Adv. Healthcare Mater., 2016, 5, 541-547.

20 K. Yue, G. Trujillo-de Santiago, M. M. Alvarez, A. Tamayol, N. Annabi and A. Khademhosseini, Biomaterials, 2015, 73, 254-271.
21 E. Hoch, T. Hirth, G. E. Tovar and K. Borchers, J. Mater. Chem. B, 2013, 1, 5675-5685.

22 E. Hoch, C. Schuh, T. Hirth, G. E. Tovar and K. Borchers, J. Mater. Sci.: Mater. Med., 2012, 23, 2607-2617.

23 C. Claaßen, M. H. Claaßen, V. Truffault, L. Sewald, T. Gem, K. Borchers and A. Southan, Biomacromolecules, 2018, 19, 42.

24 N. Celikkin, S. Mastrogiacomo, J. Jaroszewicz, X. F. Walboomers and W. Swieszkowski, J. Biomed. Mater. Res., Part A, 2018, 106, 201-209.

25 X. Li, S. Chen, J. Li, X. Wang, J. Zhang, N. Kawazoe and G. Chen, Polymers, 2016, 8, 269.

26 A. A. Abdel-Wahab, K. Alam and V. V. Silberschmidt, J. Mech. Behav. Biomed. Mater., 2011, 4, 807-820.

27 M. T. I. Mredha, N. Kitamura, T. Nonoyama, S. Wada, K. Goto, X. Zhang, T. Nakajima, T. Kurokawa, Y. Takagi and K. Yasuda, Biomaterials, 2017, 132, 85-95.

28 D. Porrelli, A. Travan, G. Turco, E. Marsich, M. Borgogna, S. Paoletti and I. Donati, Macromol. Mater. Eng., 2015, 300, 989-1000.

29 M. K. Jaiswal, J. R. Xavier, J. K. Carrow, P. Desai, D. Alge and A. K. Gaharwar, ACS Nano, 2015, 10, 246.

30 D. N. Heo, W.-K. Ko, M. S. Bae, J. B. Lee, D.-W. Lee, W. Byun, C. H. Lee, E.-C. Kim, B.-Y. Jung and I. K. Kwon, J. Mater. Chem. B, 2014, 2, 1584-1593.

31 J. R. Xavier, T. Thakur, P. Desai, M. K. Jaiswal, N. Sears, E. Cosgriff-Hernandez, R. Kaunas and A. K. Gaharwar, ACS Nano, 2015, 9, 3109-3118.

32 A. Paul, V. Manoharan, D. Krafft, A. Assmann, J. A. Uquillas, S. R. Shin, A. Hasan, M. A. Hussain, A. Memic and A. K. Gaharwar, J. Mater. Chem. B, 2016, 4, 3544-3554.

33 T. Xin, Y. Gu, R. Cheng, J. Tang, Z. Sun, W. Cui and L. Chen, ACS Appl. Mater. Interfaces, 2017, 9, 41168-41180.

34 Y. Cui, T. Zhu, A. Li, B. Liu, Z. Cui, Y. Qiao, Y. Tian and D. Qiu, ACS Appl. Mater. Interfaces, 2018, 10, 6956-6964.

35 Y. Zuo, X. Liu, D. Wei, J. Sun, W. Xiao, H. Zhao, L. Guo, Q. Wei, H. Fan and X. Zhang, ACS Appl. Mater. Interfaces, 2015, 7, 10386-10394.

36 T. Thakur, J. R. Xavier, L. Cross, M. K. Jaiswal, E. Mondragon, R. Kaunas and A. K. Gaharwar, J. Biomed. Mater. Res., Part A, 2016, 104, 879-888.

37 X. Zhou, M. Nowicki, H. Cui, W. Zhu, X. Fang, S. Miao, S. J. Lee, M. Keidar and L. G. Zhang, Carbon, 2017, 116, 615-624.

38 H. Cheng, R. Chabok, X. Guan, A. Chawla, Y. Li, A. Khademhosseini and H. L. Jang, Acta Biomater., 2018, 69, 342-351.

39 D. N. Heo, N. J. Castro, S. J. Lee, H. Noh, W. Zhu and L. G. Zhang, Nanoscale, 2017, 9, 5055-5062.

40 J. Visser, D. Gawlitta, K. E. Benders, S. M. Toma, B. Pouran, P. R. van Weeren, W. J. Dhert and J. Malda, Biomaterials, 2015, 37, 174-182.

41 K. Huang, J. Wu and Z. Gu, ACS Appl. Mater. Interfaces, 2018, 11, 2908-2916.

$42 \mathrm{~K} . \mathrm{Hu}$ and B. R. Olsen, Bone, 2016, 91, 30-38.

43 J. E. Samorezov, E. B. Headley, C. R. Everett and E. Alsberg, J. Biomed. Mater. Res., Part A, 2016, 104, 1387-1397. 
44 S. P. Parthiban, D. Rana, E. Jabbari, N. Benkirane-Jessel and M. Ramalingam, Acta Biomater., 2017, 51, 330-340.

45 X. Zhao, S. Liu, L. Yildirimer, H. Zhao, R. Ding, H. Wang, W. Cui and D. Weitz, Adv. Funct. Mater., 2016, 26, 2809-2819.

46 M. Kazemzadeh-Narbat, J. Rouwkema, N. Annabi, H. Cheng, M. Ghaderi, B. H. Cha, M. Aparnathi, A. Khalilpour, B. Byambaa and E. Jabbari, Adv. Healthcare Mater., 2017, 6, 1601122.

47 B. Byambaa, N. Annabi, K. Yue, G. Trujillo-de Santiago, M. M. Alvarez, W. Jia, M. Kazemzadeh-Narbat, S. R. Shin, A. Tamayol and A. Khademhosseini, Adv. Healthcare Mater., 2017, 6, 1700015.

48 C. Hao, Y. Kan, M. Kazemzadehnarbat, Y. Liu, A. Khalilpour, B. Li, S. Z. Yu, N. Annabi and A. Khademhosseini, ACS Appl. Mater. Interfaces, 2017, 9, 11428.

49 D. M. Gibbs, C. R. Black, J. I. Dawson and R. O. Oreffo, J. Tissue Eng. Regener. Med., 2016, 10, 187-198.
50 S. Gao, G. Tang, D. Hua, R. Xiong, J. Han, S. Jiang, Q. Zhang and C. Huang, J. Mater. Chem. B, 2019, 7, 709-729.

51 A. V. Do, B. Khorsand, S. M. Geary and A. K. Salem, Adv. Healthcare Mater., 2015, 4, 1742-1762.

52 X. Shi, S. Ostrovidov, Y. Zhao, X. Liang, M. Kasuya, K. Kurihara, K. Nakajima, H. Bae, H. $\mathrm{Wu}$ and A. Khademhosseini, Adv. Funct. Mater., 2015, 25, 2250-2259.

53 H. Xu, S. Jiang, C. Ding, Y. Zhu, J. Li and H. Hou, Mater. Lett., 2017, 201, 82-84.

54 X. Wang, M. Jiang, Z. Zhou, J. Gou and D. Hui, Composites, Part B, 2017, 110, 442-458.

55 Q. Gao, X. Niu, L. Shao, L.-y. Zhou, Z. Lin, A. Sun, J. Fu, Z. Chen, J. Hu and Y. Liu, Biofabrication, 2019, 11, 035006.

56 C. Colosi, S. R. Shin, V. Manoharan, S. Massa, M. Costantini, A. Barbetta, M. R. Dokmeci, M. Dentini and A. Khademhosseini, Adv. Mater., 2016, 28, 677-684.

57 J. Yin, M. Yan, Y. Wang, J. Fu and H. Suo, ACS Appl. Mater. Interfaces, 2018, 10, 6849-6857. 